\title{
Contenidos inmersivos violentos: investigación con eye tracking en jóvenes universitarios en España y Portugal
}

\section{Immersive content with violence: A research using eye tracking with university students in Spain and Portugal}

\author{
Luis Mañas-Viniegra; Ana-Isabel Veloso; Javier Sierra-Sánchez
}

Cómo citar este artículo:

Mañas-Viniegra, Luis; Veloso, Ana-Isabel; Sierra-Sánchez, Javier (2020). “Contenidos inmersivos violentos: investigación con eye tracking en jóvenes universitarios en España y Portugal". El profesional de la información, v. 29, n. 1, e290108.

https://doi.org/10.3145/epi.2020.ene.08

Artículo recibido el 10-04-2019 Aceptación definitiva: 25-06-2019

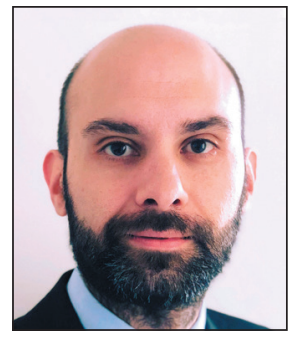

Luis Mañas-Viniegra $\bowtie$ http://orcid.org/0000-0001-9129-5673

Universidad Complutense de Madrid Facultad de Ciencias de la Información Avda. Complutense, 3 28040 Madrid, España Imanas@ucm.es

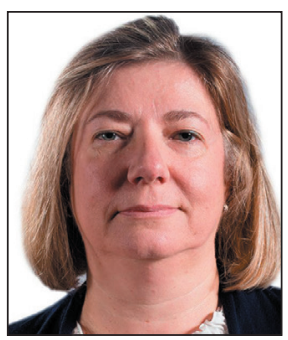

Ana-Isabel Veloso http://orcid.org/0000-0002-5070-0756

Universidade de Aveiro Departamento de Comunicação e Arte DigiMedia

Campus universitário de Santiago. 3810-193 Aveiro, Portugal aiv@ua.pt

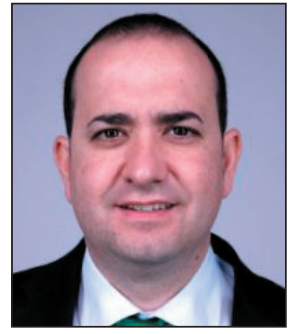

Javier Sierra-Sánchez https://orcid.org/0000-0001-8572-7564

Universidad Complutense de Madrid Facultad de Ciencias de la Información Avda. Complutense, 3 28040 Madrid, España javiersierrasanchez@pdi.ucm.es

\section{Resumen}

Los diarios se han visto afectados por una progresiva pérdida de lectores durante la última década, específicamente entre los menores de 24 años. Éstos continúan informándose sobre sus áreas de interés, pero en redes sociales. Por ello, la prensa ha incorporado un periodismo lúdico, inmersivo y participativo a partir de tecnologías como la realidad virtual o los vídeos $360^{\circ}$. Mediante un análisis cuantitativo y cualitativo de los datos obtenidos con la técnica de eye tracking y la realización posterior de entrevistas semiestructuradas, esta investigación comprueba la atención que los universitarios españoles y portugueses prestan a los contenidos periodísticos inmersivos violentos. También se analiza la comprensión y la capacidad de concienciación de los estudiantes ante este material informativo con hechos violentos. Se pone de manifiesto la capacidad del periodismo inmersivo para atraer la atención del público menor de 24 años y para concienciar sobre conflictos sociales a partir de la visualización de contenidos violentos.

Financiación

Proyecto financiado por el programa Young State de la Cátedra TMKF de Comunicación y Marketing por la Infancia, Adolescencia y Juventud de la Universidad Complutense de Madrid (YS-009/18). 


\title{
Palabras clave
}

Periodismo digital; Periodismo inmersivo; Serious games; Juegos serios; Violencia; Realidad virtual; Vídeo $360^{\circ}$; Estudios de usuarios; UX (user experience); Jóvenes; Eye tracking; España; Portugal.

\begin{abstract}
Newspapers have been affected by a progressive loss of readership over the last decade, especially among those under 24 years old. This group continues to stay informed about topics of their interest, but through social networks. For this reason, the press has incorporated a playful, immersive, and participatory journalism based on technologies such as virtual reality or 360 videos. Through quantitative and qualitative analysis of data obtained from eye tracking, and by subsequently performing semi-structured interviews, this research verifies the attention paid by Spanish and Portuguese university students to violent, immersive, journalistic content. Students' understanding and awareness of this informative material with violent events is analyzed. The capability of immersive journalism to capture the attention of the public under 24 years old and to raise awareness about social problems based on visualization of violent content is highlighted.
\end{abstract}

\section{Keywords}

Digital journalism; Immersive journalism; Serious games; Violence; Virtual reality; VR; 360 video; Users studies; UX (user experience); Young people; Eye tracking; Spain; Portugal.

\section{Introducción}

La lectura de los periódicos se ha resentido notablemente por el efecto del consumo mediático online. El Estudio General de Medios (AIMC, 2018) ya sitúa a los diarios como el sexto medio por penetración en España tras diez años de permanente caída, lo que supone que su consumo fue en 2018 el 57,48\% del que tenía en 2008 . El menor dato de consumo se encuentra en los jóvenes de 14 a 19 años (3,9\%) y en los de 20 a 24 años (4,3\%). A pesar de que el 43,5\% de los jóvenes afirma no leer nunca, el $46,1 \%$ usa internet todos los días para obtener información sobre asuntos sociales o políticos, frente al 36,6\% de media en todos los perfiles de edad (CIS, 2017), lo que a priori refleja interés sobre la actualidad informativa online y puede suponer una oportunidad para revitalizar los periódicos con géneros emergentes y formatos que proporcionen algún nivel de juego e interacción social.

La gamificación o ludificación es la aplicación de los juegos a un contexto ajeno a su finalidad original, para fidelizar, incentivar la acción, fomentar el aprendizaje o promover la resolución de cuestiones, partiendo del pensamiento lúdico que subyace en los juegos para trascender el mero entretenimiento (Deterding et al., 2011). La gamificación ha encontrado en la recreación y simulación de realidades o los videojuegos posibilidades interactivas e inmersivas plenamente integradas en la esfera académica e investigadora (Scolari, 2013) y estos juegos han pasado a denominarse serious games (Girard; Ecalle; Magnan, 2013).

Los juegos serios presentan un carácter pedagógico basado en un entretenimiento que favorece una inmersión en la realidad con un punto de vista determinado (Ulrich; Helms, 2017), sin olvidar que su narrativa puede resultar apropiada para áreas como la Psicología, el Periodismo o la Comunicación digital (Lugmayr et al., 2017), contribuyendo a concienciar sobre comportamientos socialmente responsables (Yam et al., 2017) y a mitigar levemente los sesgos cognitivos, permitiendo mejorar la empatía y en definitiva el cambio social (Papoutsi; Drigas, 2016).

Si bien pudieran derivarse efectos perniciosos de adicción o agresividad, algunos autores mantienen que éstos han sido exagerados en la misma proporción que en los videojuegos en general (Bosche; Kattner, 2013), frente a los que encuentran correlación entre la exposición a los videojuegos violentos y una mayor agresividad en el comportamiento (Anderson, 2004) y otros que inciden en los síntomas depresivos y los rasgos antisociales previos como predictores de la agresividad y la violencia (Ferguson, 2011).

Los juegos serios en el área periodística y de la comunicación digital comienzan a partir de los denominados newsgames (Frasca, 2007), género iniciado por The New York Times al incorporar videojuegos generados a partir de algún acontecimiento informativo en su edición online como complemento al contenido de las noticias publicadas, a pesar de que la bibliografía científica ha prestado más atención a los aspectos lúdicos que a los informativos y comunicativos (Gómez-García; Navarro-Sierra, 2013) y a que muchos de ellos se generan para trasmitir una opinión (Gómez-García; Cabeza-San-Deogracias, 2016).

Al potenciar información y opinión sobre entretenimiento se evoluciona hacia un género más próximo al reportaje y el documental informativo y menos al videojuego. Es la adaptación de los newsgames a un periodismo lúdico, inmersivo y participativo (García-Ortega; García-Avilés, 2018) a partir de la realidad aumentada, virtual o el vídeo $360^{\circ}$ para representar de forma interactiva parte de una realidad. Este género se ha denominado periodismo inmersivo (Domínguez-Martín, 2015) o docugame

\author{
Al potenciar información y opinión so- \\ bre entretenimiento se evoluciona hacia \\ un género más próximo al reportaje y el \\ documental informativo y menos al vi- \\ deojuego
}


(Vázquez-Herrero; López-García, 2016; 2017) y obedece a una evolución de los medios informativos digitales que han afrontado un proceso de convergencia (Jenkins, 2006), posibilitando al público convertirse en protagonista -o al menos en observador privilegiado- de la actualidad al encontrarse inmerso en ella decidiendo qué punto de vista desea adoptar dentro del camino que le guía a través de los acontecimientos.

El periodismo inmersivo ha sido posible gracias a la integración de periodistas y programadores en los procesos tecnológicos de los periódicos digitales (Marcos-Recio; Edo-Bolós; Parra Valcarce, 2018). Se ha revelado como un método para mejorar la visibilidad de los colectivos sin voz en los medios, proporcionando perspectivas alternativas sobre los hechos y mejorando la concienciación de los públicos (Sacco; Gorin; Schiau, 2018), ya que la vivencia narrativa mejora la comprensión del acontecimiento y la empatía hacia sus protagonistas (Ruiz-Collantes, 2008). Tanto el periodismo inmersivo en formato de realidad virtual como en vídeo $360^{\circ}$ acercan la realidad al espectador (Pérez-Seijo, 2017), mejoran la credibilidad de la fuente, la empatía y la intención de compartir más el contenido que en el formato tradicional en texto e imágenes (Sundar; Kang; Oprean, 2017). Además posibilitan la exploración del lugar de los hechos, experimentando los públicos la sensación de presencia y participación de la actualidad informativa (Domínguez-Martín, 2015), lo que ha motivado que The New York Times haya producido 300 reportajes inmersivos en el trienio 2015-2017 (Benítez-de-Gracia; Herrera-Damas, 2018) y se haya convertido en el principal impulsor de este gé-

El periodismo inmersivo en formato de realidad virtual o de vídeo $360^{\circ}$ acerca la realidad al espectador y mejora la empatía y la intención de compartir más el contenido que el formato tradicional nero periodístico. La interacción alcanzada oscila desde lo selectivo, si el público influye sobre el relato final; pasando por lo inmersivo, formando parte del relato; lo social, facilitando al público expresarse sobre el asunto; lo generativo, si posibilita al receptor producir contenidos; hasta lo físico, si se alcanza una plena experiencia personal a través de la realidad aumentada o la virtual (Vázquez-Herrero; Negreira-Rey; Pereira-Fariña, 2017).

En definitiva, la convergencia digital de estos géneros emergentes y formatos informativos en múltiples soportes mediáticos, que se ha venido a denominar periodismo transmedia (Jenkins, 2003; Scolari, 2009), proporciona una experiencia inmersiva lúdico-periodística en la que es necesario determinar si favorece una mayor atención del público más joven hacia la actualidad informativa con contenido violento y si además la comprende, participa de ella y forma una opinión sobre el acontecimiento.

\section{Objetivo y método}

La finalidad de esta investigación es comprobar la atención prestada por los jóvenes universitarios de España y Portugal a los contenidos periodísticos inmersivos violentos, así como la comprensión de los propios contenidos informativos en ausencia del género periodístico de la noticia tradicional. Para la consecución de este objetivo general, se utiliza la técnica de eye tracking para establecer a qué áreas de interés presta atención el público, así como la intensidad y reiteración de la misma, de modo que sea posible diferenciar si la audiencia centra la atención en los agresores o la agresión en sí, las víctimas o además su atención visual busca ayuda en quienes presencian la escena violenta.

Los objetivos específicos son:

- Determinar si el perfil de audiencia menos afín a los periódicos presta atención al periodismo inmersivo y a la noticia que amplía tal contenido.

- Establecer diferencias en la atención en el público español con respecto al portugués.

- Diferenciar el grado de atención mostrado hacia el agresor o la agresión con respecto a las víctimas y a los testigos del acontecimiento periodístico.

- Verificar el grado de comprensión del contenido de la información transmitido.

La neurocomunicación (Cuesta-Cambra; Niño-González; Rodríguez-Terceño, 2017) es una combinación de la Psicología, la Neurociencia y la Economía (Madan, 2010) que hace posible la medición del proceso cognitivo de los sujetos sobre determinados estímulos. Esta especialidad solventa las limitaciones que presentan las tradicionales encuestas, grupos de discusión o entrevistas en profundidad a la hora de que los individuos informen conscientemente sobre sus comportamientos, motivaciones o emociones (Ariely; Berns, 2010). Dentro de la neurocomunicación, el eye tracking es una técnica biométrica capaz de medir la atención visual a partir de los movimientos y fijaciones oculares hacia las zonas de interés (Duchowski, 2013). Es precisamente la atención la que inicia el procesamiento cognitivo y afectivo y numerosos autores han puesto de manifiesto que hay una correlación positiva entre la atención y la memoria (Loftus; Kallman, 1979; Bornstein; D’Agostino, 1992; Goodrich, 2011). Para la presente investigación se ha utilizado un eye tracker TOBII X3-120 en Portugal y un X2-60 en España.

Los estímulos presentados a los sujetos fueron dos vídeos $360^{\circ}$ que reconstruyen con modelado 3D los hechos que constituyeron noticia e incorporan el sonido real grabado 
En la investigación han participado voluntariamente 120 individuos -entre junio y diciembre de 2018- con el perfil que la bibliografía científica señalaba mostrar un menor interés hacia las noticias tradicionales en prensa, a pesar de utilizar las redes sociales para informarse: jóvenes de entre 18 y 24 años, que en la investigación proceden de las universidades Complutense y Rey Juan Carlos de Madrid en España (50\%-grupo 1) y la Universidad de Aveiro (50\%-grupo 2) en Portugal. Ambos grupos registraron paridad de género, a pesar de que éste no manifestó diferencias significativas.

Los estímulos presentados a los sujetos fueron dos vídeos $360^{\circ}$ creados por Emblematic Group, reconstruyendo con modelado 3D los hechos que constituyeron noticia e incorporando el sonido real grabado.

El primero, Use offorce, recrea con realidad virtual la muerte de Anastasio Hernández en la frontera entre México y EUA tras ser golpeado por agentes.

El segundo se titula Kiya y recrea una escena de violencia de género que finaliza con el asesinato de la mujer maltratada.

En los dos casos se busca concienciar al convertir al espectador en uno de los testigos y el audio es el real de las grabaciones recogidas en las respectivas escenas de los crímenes. Para ambos estímulos (tabla 1) se han definido áreas de interés (AOI) dinámicas, que consiguen registrar la atención en imágenes en movimiento y comparar la atención que se presta a los agresores, las víctimas, la ayuda que puedan proporcionar los testigos presentes en la escena o que puedan entrar al rescate por una puerta. Todo ello en los momentos de máxima tensión en cada uno de los casos: una visión directa de la agresión, el momento en el que el agresor muestra mayor nerviosismo con el arma en la mano, cuando apunta en la cabeza con la pistola a la víctima y al sonar el primer disparo.

Tabla 1. Áreas de interés (AOI) de los estímulos Use of force y Kiya

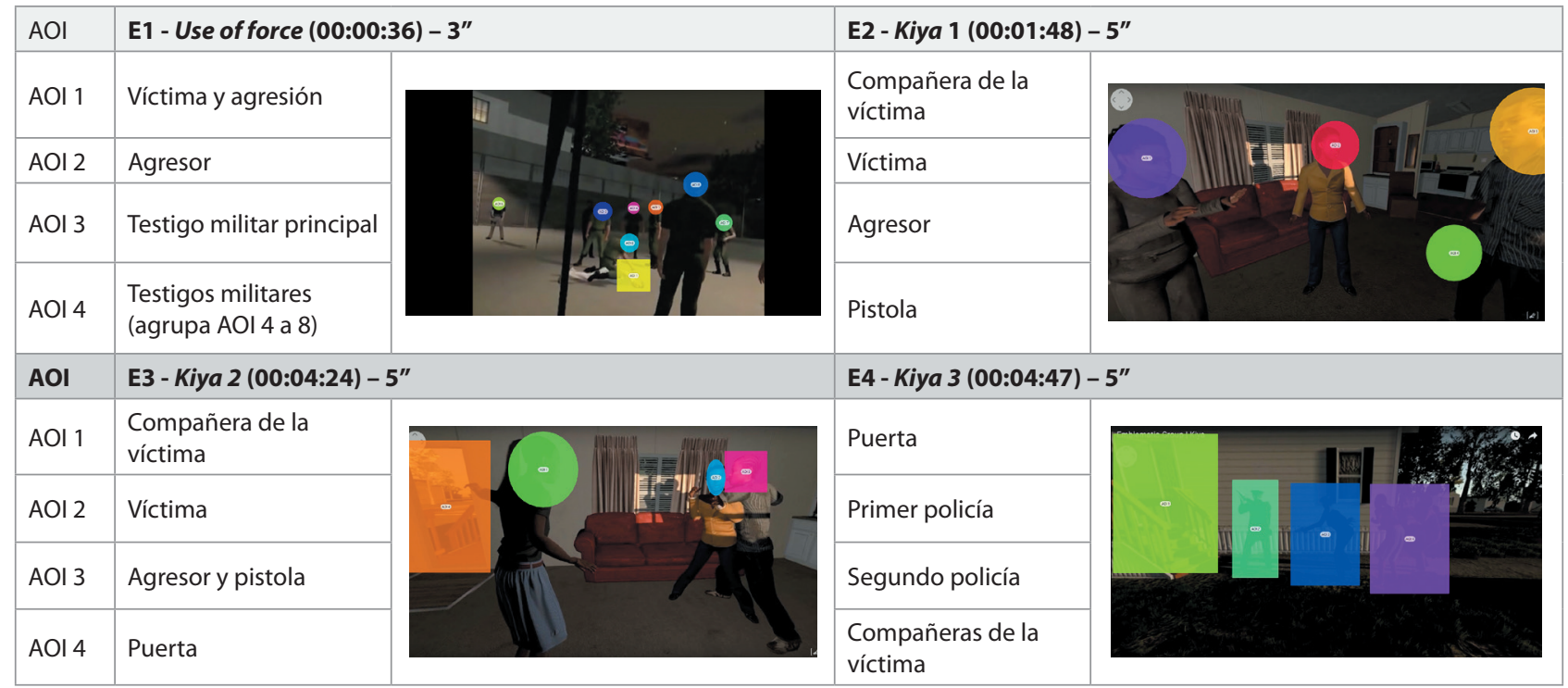

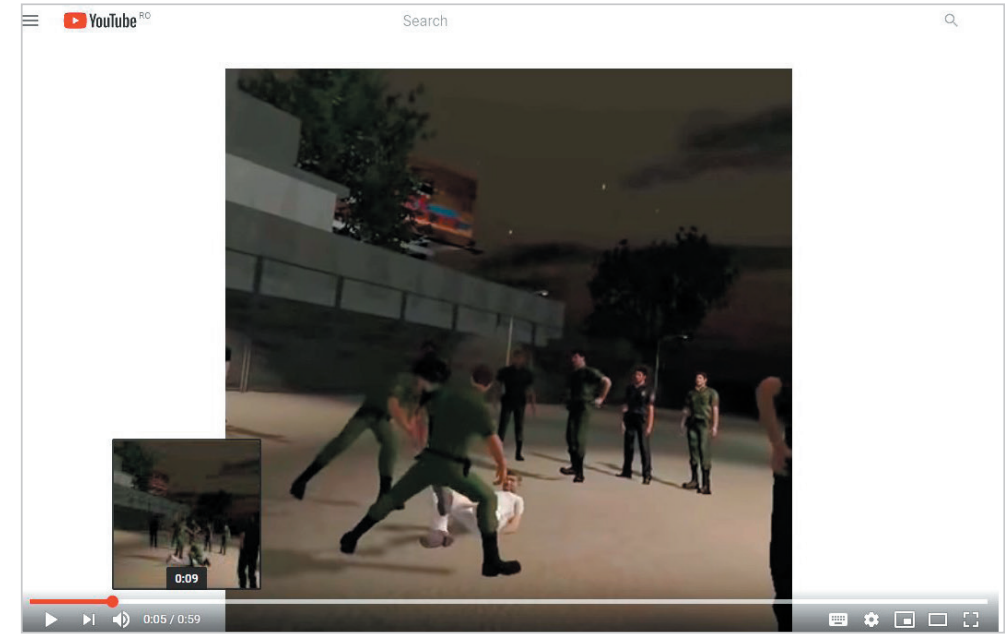

Escena de Use of force

https://www.youtube.com/watch?v=I1QM-9BRU_4

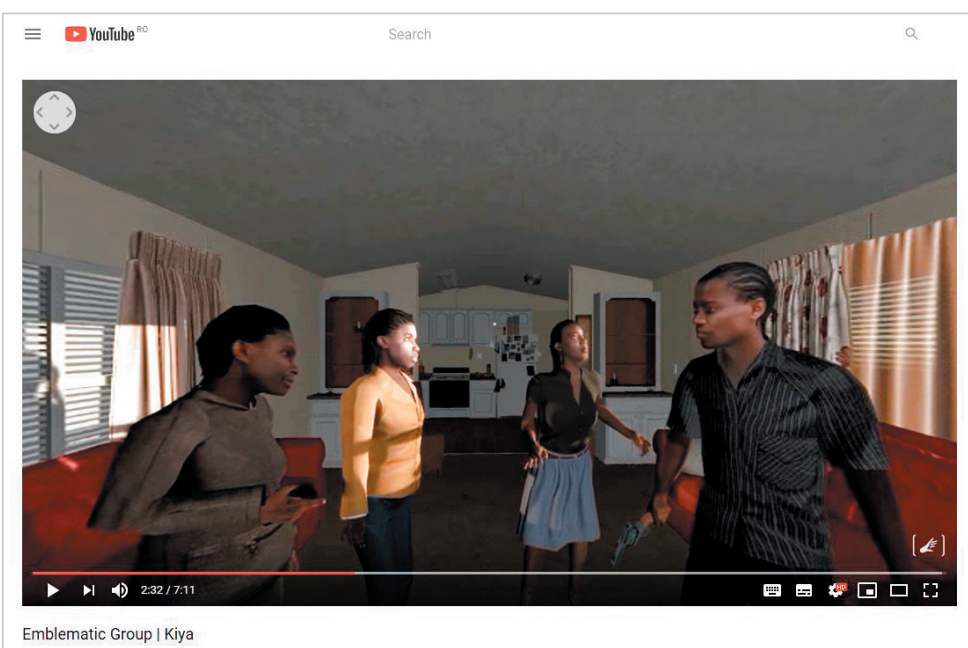

Escena de Kiya

https://www youtube.com/watch?v=qYsAlukRqog 
La variable dependiente se localiza en los niveles de atención registrados en los estímulos presentados, mientras que las variables independientes son la procedencia de España o Portugal de los sujetos y el género, puesto que todos los participantes se encuentran en un rango de edad único elegido por los motivos aludidos anteriormente. Se evalúan cualitativamente los mapas de calor o heat maps y cuantitativamente:

- cantidad de fijaciones oculares o fixation count (FC);

- duración en segundos de cada fijación o fixation duration (FD);

- segundos que transcurren desde que el estímulo hace acto de presencia hasta que se produce la primera fijación o time from fixation (TFF);

- duración total en segundos de atención o total fixation duration (TFD).

El software estadístico con el que se han tratado los datos obtenidos ha sido SPSS v.25.

Tras finalizar la visualización de todos los estímulos, se realizaron entrevistas semiestructuradas entre los 120 sujetos para dilucidar qué hecho informativo consideraban que se había producido a partir del vídeo que acababan de visualizar, de modo que se valorase la comprensión del propio contenido como complementario o sustitutivo de los géneros periodísticos tradicionales, teniendo la posibilidad de visualizar la noticia asociada posteriormente.

\section{Resultados y discusión}

El análisis cualitativo de los heat maps pone de manifiesto (tabla 2) que la atención de los participantes se centra en la acción violenta, ya que ésta se produce en todos los sujetos con independencia de si se encuentra más próxima al agresor o a la víctima. Sin embargo, se aprecian diferencias entre ambos grupos. El grupo 2 presta atención más repartida entre la víctima y el agresor y, además, busca ayuda más focalizada al registrar mayor atención a los testigos que podrían intervenir para detener la acción y la puerta por la que podrían buscar una salida o esperar la entrada de otro tipo de ayuda.

Tabla 2. Heat maps de los estímulos Use of force y Kiya

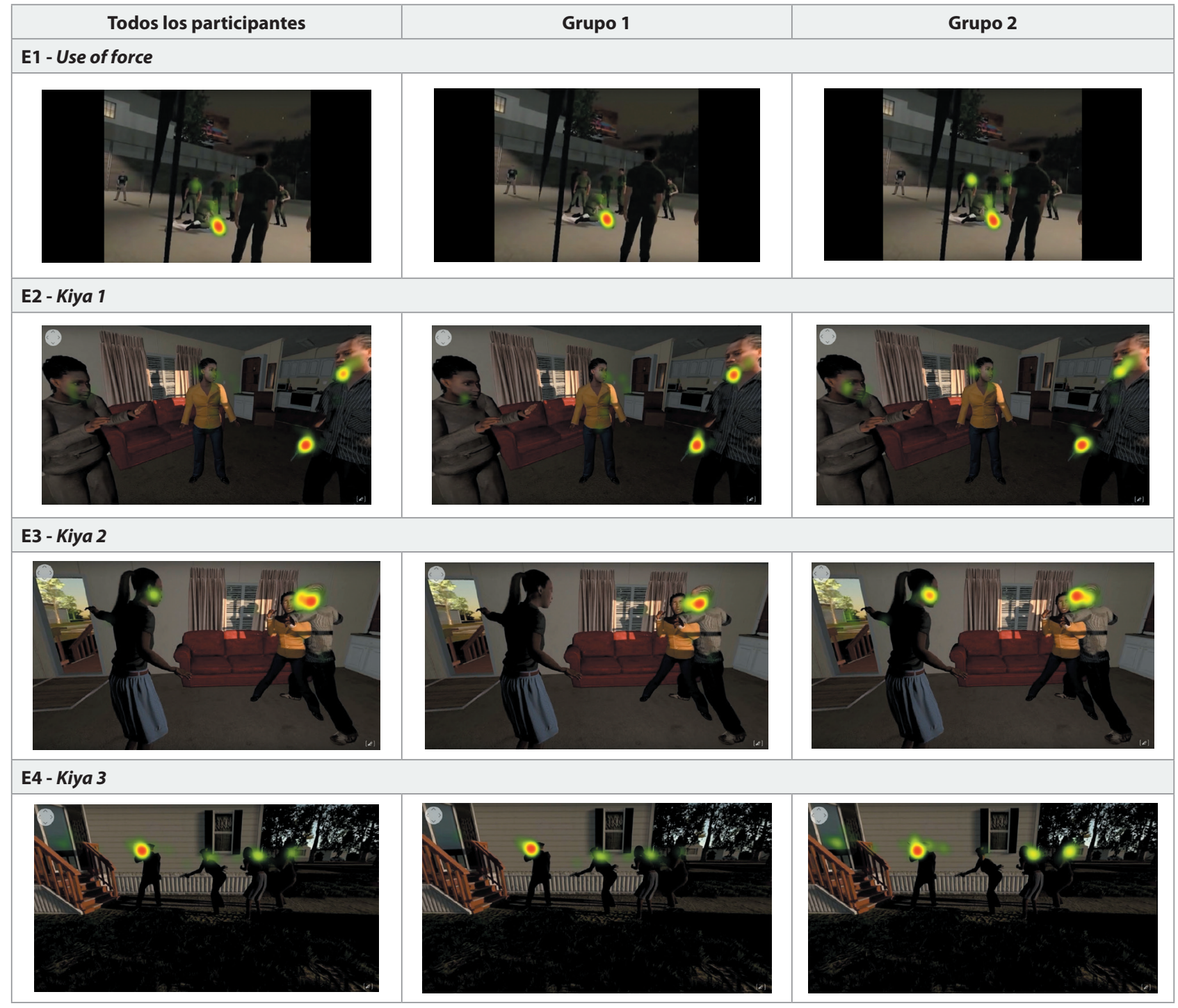


En los tres estímulos en los que se ve en pantalla la agresión, ya sea en forma de golpes o de amenaza con la pistola, se obtiene la atención del $100 \%$ de los sujetos. La atención prestada a la víctima oscila, en función de los estímulos, desde el $100 \%$-E1, en la que no se puede separar de la agresión por la mínima distancia entre ambas zonas-y el $84,20 \%$ (E2) y el $50 \%$ (E3), a pesar de superar en atención al agresor, que oscila entre el 35,80\% (E3), $69,20 \%$ (E1) y $100 \%$ (E2). La escena de máxima violencia

(E4), aunque ésta no sea visible directamente, acapara la mayor atención, con el $100 \%$ de los participantes en tres de las cuatro AOI. Los testigos secundarios en E1-Use of force acaparan una mínima atención, por lo que se excluyen del análisis final.

En la comparativa realizada entre las áreas de interés similares entre todos los sujetos, las diferencias son claramente significativas $(p<0,05)$. Al comparar la atención prestada a la víctima o a sus acompañantes, la primera presenta un menor TFF en todos los casos en los que está acompañada, destacando su presencia conjunta con la agresión (TFF=0,88; $p=0,000)$, mientras que la atención más rápida la consigue una de las acompañantes de la víctima en el momento de mayor violencia, cuando la propia víctima no se encuentra presente en la imagen (TFF=0,31; $p=0,000)$. Estas dos situaciones también registran un mayor número de fijaciones oculares significativo ( $F C=3,01$ y $F C=4,04$, respectivamente; $p=0,000$ ) y la mayor duración media de cada fijación ocular en el caso del primer caso ( $F D=0,46 ; p=0,000)$.

El agresor registra la mayor atención en el momento de máxima tensión con el arma con la que se produce la agresión, con la fijación media más rápida (TFF=0,43, $p=0,000)$, el mayor número medio de fijaciones $(F D=10,48 ; p=0,000), y$ la segunda mayor duración media de cada una de esas fijaciones ( $F D=0,035 ; p=0,000)$.

En cuanto a la presencia de testigos compañeras de la víctima, policías que acuden al rescate o la entrada/salida a través de la cual el espectador busca la llegada de alguna ayuda o una salida a la situación violenta, la atención más rápida la registran los policías que activamente acuden a socorrer a la víctima (TFF=0,60: $p=0,000)$.

De igual modo, el policía que acude recibe un mayor número de fijaciones oculares ( $F C=6 ; p=0,000)$ con la segunda mayor duración media en cada fijación ( $F D=0,39 ; p=0,000$ ).

La puerta de entrada registra el segundo mayor número de fijaciones oculares ( $F C=2,69 ; p=0,000$ ) junto a la de salida -en función del plano de la grabación- $(F C=2,16 ; p=0,000)$, aunque con menor duración media en cada fijación ( $F D=0,28$ y $F D=0,25$, respectivamente; $p=0,000)$ que el policía, los testigos de la agresión en Use of force $(F D=0,41 ; p=0,000)$ o la acompañante de Kiya durante la agresión ( $F D=0,29 ; p=0,000)$.

Las diferencias significativas entre ambos grupos (tabla 2) ponen de manifiesto que en E1 el grupo 2 presta atención más rápidamente al agresor ( $T F F=0,84 ; p=0,001)$, con un mayor número de fijaciones de media $(F C=1,70 ; p=0,010)$ que el grupo 1 , a pesar de que éste mantiene la atención durante más tiempo en cada fijación ( $F D=0,44 ; p=0,001)$. El grupo 1 , además, muestra un mayor número de fijaciones a la víctima ( $F C=3,35 ; p=0,006)$, con mayor duración en cada fijación $(F D=0,54 ; p=0,000)$.

En E2 también se aprecia que el grupo 2 presta atención antes al agresor (TFF=0,70; $p=0,000)$, con un mayor número de fijaciones oculares $(F C=5,82 ; p=0,024)$ y también de manera significativa con respecto al grupo 1 , hace lo propio con la compañera de la víctima ( $T F F=2,19 ; p=0,005 ; F C=2,28 ; p=0,000)$. El grupo 1 en cambio, dirige antes su atención a la víctima (TFF=1,10; $p=0,020)$ con una mayor duración media ( $F D=0,25 ; p=0,000)$.

A pesar de que en E3 las diferencias significativas entre ambos grupos son puntuales, en E4 sí se observa que el grupo 1 busca ayuda con la mirada, fijándose antes en la compañera de la víctima (TFF=0,20; $p=0,000)$ y la policía (TFF=0,58; $p=0,033$ ), destinando a ésta un mayor número de fijaciones ( $F C=2,47 ; p=0,050)$ y una mayor duración de cada fijación al segundo policía ( $F D=0,44$; $\mathrm{p}=0,001)$.

El 69,17\% de los participantes (desviación de 0,464) pudo describir el contenido general de la información en el caso de Use of force y el 86,67\% (desviación de 0,341) en el caso de Kiya, a pesar de que los detalles sobre el cuándo, dónde y por qué de la noticia no se concretan en ningún caso, lo que sugiere la necesidad de complementar estos vídeos $360^{\circ}$ de periodismo inmersivo con una información que los complete. El interés en buscar la noticia que explica el caso de Kiya fue notablemente superior (74,17\%; desviación de 0,395) al de Use of force $(19,17 \%$; desviación de 0,440$)$ y parece que "su mayor realismo", una mayor "claridad de lo que está pasando" y la "violencia", "injusticia" o "rabia" sentida ante la 
Tabla 2. Kruskal Wallis test entre grupos de participantes

\begin{tabular}{|c|c|c|c|c|c|c|c|}
\hline \multicolumn{4}{|c|}{ E1 - Use of force } & \multicolumn{4}{|c|}{ E2 - Kiya 1} \\
\hline \multirow{2}{*}{$\mathrm{AOI}$} & \multicolumn{2}{|c|}{ Media FC (No) } & \multirow{2}{*}{ P-value } & \multirow{2}{*}{$\mathrm{AOI}$} & \multicolumn{2}{|c|}{ Media FC ( $\left.\mathrm{N}^{\circ}\right)$} & \multirow{2}{*}{ P-value } \\
\hline & Grupo 1 & Grupo 2 & & & Grupo 1 & Grupo 2 & \\
\hline AOI 1 & 3,35 & 2,67 & ${ }^{*} 0,006$ & AOI 1 & 1,67 & 2,28 & ${ }^{*} 0,000$ \\
\hline AOI 2 & 1,24 & 1,70 & ${ }^{*} 0,010$ & AOI 2 & 2,20 & 2,66 & 0,191 \\
\hline AOI 3 & 1,50 & 1,43 & 0,916 & AOI 3 & 5,15 & 5,82 & ${ }^{*} 0,024$ \\
\hline $\mathrm{AOI} 4$ & ND & ND & ND & $\mathrm{AOI} 4$ & 4,32 & 4,23 & 0,733 \\
\hline \multirow{2}{*}{ AOI } & \multicolumn{2}{|c|}{ Media FD (“) } & \multirow{2}{*}{ P-value } & \multirow{2}{*}{$\mathrm{AOI}$} & \multicolumn{2}{|c|}{ Media FD (") } & \multirow{2}{*}{ P-value } \\
\hline & Grupo 1 & Grupo 2 & & & Grupo 1 & Grupo 2 & \\
\hline AOI 1 & 0,54 & 0,39 & $* 0,000$ & AOI 1 & 0,33 & 0,28 & 0,165 \\
\hline AOI 2 & 0,44 & 0,30 & ${ }^{*} 0,001$ & AOI 2 & 0,25 & 0,18 & ${ }^{*} 0,000$ \\
\hline $\mathrm{AOI} 3$ & 0,38 & 0,43 & 0,079 & $\mathrm{AOI} 3$ & 0,28 & 0,23 & ${ }^{*} 0,015$ \\
\hline $\mathrm{AOI} 4$ & ND & ND & ND & $\mathrm{AOI} 4$ & 0,27 & 0,25 & 0,097 \\
\hline \multirow{2}{*}{$\mathrm{AOI}$} & \multicolumn{2}{|c|}{ Media TTF (") } & \multirow{2}{*}{ P-value } & \multirow{2}{*}{ AOI } & \multicolumn{2}{|c|}{ Media TTF (") } & \multirow{2}{*}{ P-value } \\
\hline & Grupo 1 & Grupo 2 & & & Grupo 1 & Grupo 2 & \\
\hline AOI 1 & 0,83 & 0,93 & 0,063 & AOI 1 & 2,58 & 2,19 & ${ }^{*} 0,005$ \\
\hline AOI 2 & 1,34 & 0,84 & ${ }^{*} 0,001$ & $\mathrm{AOI} 2$ & 1,10 & 1,90 & ${ }^{*} 0,020$ \\
\hline AOI 3 & 2,41 & 2,05 & 0,214 & $\mathrm{AOI} 3$ & 0,94 & 0,70 & ${ }^{*} 0,000$ \\
\hline $\mathrm{AOI} 4$ & ND & ND & ND & $\mathrm{AOI} 4$ & 1,14 & 1,35 & 0,252 \\
\hline \multicolumn{4}{|c|}{ E3 - Kiya 2} & \multicolumn{4}{|c|}{ E4 - Kiya 3} \\
\hline \multirow{2}{*}{$\mathrm{AOI}$} & \multicolumn{2}{|c|}{ Media FC $\left(\mathrm{N}^{\circ}\right)$} & \multirow{2}{*}{ P-value } & \multirow{2}{*}{$\mathrm{AOI}$} & & No) & \\
\hline & Grupo 1 & Grupo 2 & & & Grupo 1 & Grupo 2 & \\
\hline AOI 1 & 1,50 & 2,25 & 0,064 & AOI 1 & 2,50 & 2,81 & 0,309 \\
\hline AOI 2 & 1,24 & 1,58 & ${ }^{*} 0,044$ & AOI 2 & 5,87 & 6,13 & 0,635 \\
\hline $\mathrm{AOI} 3$ & 10,45 & 10,52 & 0,876 & AOI 3 & 2,47 & 1,95 & $* 0,050$ \\
\hline $\mathrm{AOI} 4$ & 2,25 & 2,13 & 0,443 & $\mathrm{AOI} 4$ & 3,80 & 4,28 & 0,241 \\
\hline P & & & $0 \quad 1$ & PO & & & \\
\hline AUI & Grupo 1 & Grupo 2 & P-value & $A \cup 1$ & Grupo 1 & Grupo 2 & P-value \\
\hline AOI 1 & 0,20 & 0,30 & ${ }^{*} 0,016$ & AOI 1 & 0,29 & 0,26 & 5,79 \\
\hline AOI 2 & 0,16 & 0,15 & 0,599 & $\mathrm{AOI} 2$ & 0,44 & 0,34 & ${ }^{*} 0,001$ \\
\hline $\mathrm{AOI} 3$ & 0,39 & 0,31 & ${ }^{*} 0,000$ & AOI 3 & 0,21 & 0,21 & 0,831 \\
\hline $\mathrm{AOI} 4$ & 0,26 & 0,24 & 0,379 & $\mathrm{AOI} 4$ & 0,24 & 0,25 & 0,557 \\
\hline P & & & & 䧑 & & $\left({ }^{(\prime)}\right.$ & \\
\hline AUI & Grupo 1 & Grupo 2 & -value & $A \cup 1$ & Grupo 1 & Grupo 2 & r-value \\
\hline AOI 1 & 1,70 & 2,63 & $* 0,050$ & AOI 1 & 2,86 & 2,62 & 0,369 \\
\hline AOI 2 & 1,89 & 2,23 & 0,300 & AOI 2 & 1,05 & 1,11 & 0,287 \\
\hline AOI 3 & 0,43 & 0,44 & 0,414 & AOI 3 & 0,58 & 0,61 & ${ }^{*} 0,033$ \\
\hline $\mathrm{AOI} 4$ & 2,10 & 1,93 & 0,712 & $\mathrm{AOI} 4$ & 0,20 & 0,42 & ${ }^{*} 0,000$ \\
\hline
\end{tabular}

experiencia inmersiva declarada por los participantes ayuda a cumplir con la finalidad de concienciar sobre el contenido violento y despierta el interés informativo para buscar ese contenido en una noticia. En último lugar, se realizó la prueba de $\mathrm{Chi}^{2}$ para comprobar las diferencias entre el mayor porcentaje de sujetos que describen el contenido general de la información a partir del vídeo y quienes buscan posteriormente $\left(\chi^{2}(2)=4,969 ; p=0,011\right)$ sobre los que no, rechazándose $\mathrm{H}_{0}$ y concluyendo que ambas variables están correlacionadas. 


\section{Conclusiones}

A pesar del continuo descenso de audiencia experimentado por los periódicos en virtud de la información que los jóvenes obtienen de internet y las redes sociales sobre las cuestiones de su interés, la investigación realizada pone de manifiesto la capacidad del periodismo inmersivo para atraer la atención de los jóvenes universitarios españoles y portugueses.

\author{
El $74,17 \%$ de los participantes decidió \\ leer la información publicada sobre el \\ hecho narrado en el vídeo $360^{\circ}$ sobre \\ violencia machista
}

Al margen del interés que aporta el periodismo inmersivo para el lector más joven, demostrado por una atención prestada por parte del $100 \%$ de los participantes a alguna de las áreas marcadas con contenido violento en cada estímulo, el $86,67 \%$ de los participantes ha sido capaz de describir el contenido general informativo del vídeo $360^{\circ}$ sobre la violencia machista, decidiendo el $74,17 \%$ acceder a la noticia publicada después de su experiencia inmersiva, superando así el enfoque lúdico de los trabajos realizados hasta el momento con los diversos tipos de juegos serios.

La capacidad de concienciación de estos contenidos inmersivos violentos era otra de las cuestiones a resolver. A pesar de que la acción violenta es la que atrae la atención principal de los participantes, alcanzando al 100\% de los sujetos, acaparando el caso de violencia machista más atención que la agresión policial y las víctimas, más que los agresores. En las escenas en las que las víctimas no se encuentran presentes directamente en la imagen, la atención a la policía, las compañeras de la víctima o la puerta de entrada/salida reflejan un intento visual de socorrer a la víctima. Por tanto, los resultados se encuentran en línea con la capacidad de mejorar la empatía, el cambio social y la defensa de los derechos humanos que la bibliografía científica asignaba a cualquier tipo de juego serio, entre los que se encontraría este tipo de periodismo inmersivo.

Las diferencias entre grupos son significativas únicamente en áreas de interés puntuales. El grupo 2 fija su atención de manera más distribuida entre la víctima, la búsqueda de ayuda y la agresión, aunque los dos grupos enfocan su atención en los momentos de máxima violencia. Sin embargo, la rapidez con la que el grupo 2 fija su atención en el agresor y el número de veces que lo hace es significativamente mayor que en el grupo 1, con una atención más intensamente centrada en la víctima y la ayuda. Estos resultados parecen sugerir una mayor concienciación e implicación por parte de los participantes españoles con las víctimas de la violencia machista, quienes han experimentado un sentimiento de injusticia y rabia.

\section{Referencias}

AIMC (2018). EGM. Abril 2017 a marzo 2018. Resumen general. https://www.aimc.es/a1mc-cOnt3nt/uploads/2018/04/resumegm118.pdf

Anderson, Craig A. (2004). "An update on the effects of playing violent video games". Journal of adolescence, v. 27, n. 1, pp. 113-122.

https://doi.org/10.1016/j.adolescence.2003.10.009

Ariely, Dan; Berns, Gregory S. (2010). "Neuromarketing: The hope and hype of neuroimaging in business". Nature reviews neuroscience, n. 11, pp. 284-292.

https://doi.org/10.1038/nrn2795

Benítez-de-Gracia, María-José; Herrera-Damas, Susana (2018). “El reportaje inmersivo en vídeo $360^{\circ}$ : diseño de un modelo de análisis". El profesional de la información, v. 27, n. 1, pp. 149-161.

https://doi.org/10.3145/epi.2018.ene.14

Bornstein, Robert F.; D’Agostino, Paul R. (1992). "Stimulus recognition and the mere exposure effect". Journal of personality and social psychology, v. 63, n. 4, pp. 545-552.

https://doi.org/10.1037/0022-3514.63.4.545

Bosche, Wolfgang; Kattner, Florian (2013). "Fear of (serious) digital games and game-based learning? Causes, consequences and a possible countermeasure". In: Felicia, Patrick. Developments in current game-based learning design and deployment. Hershey, PA: IGI Global, pp. 203-218. ISBN: 9781455518640

https://doi.org/10.4018/978-1-4666-1864-0.ch015

CIS (2017). Barómetro de noviembre 2017. Centro de Investigaciones Sociológicas. http://www.cis.es/cis/opencm/ES/1_encuestas/estudios/ver.jsp?estudio=14370

Cuesta-Cambra, Ubaldo; Niño-González, José-Ignacio; Rodríguez-Terceño, José (2017). “El procesamiento cognitive en una app educativa con electroencefalograma y eye tracking". Comunicar, v. 52, n. 3, pp. 41-50.

https://doi.org/10.3916/C52-2017-04 
Deterding, Sebastian; Khaled, Rilla; Nacke, Lennart E.; Dixon, Dan (2011). “Gamification: Toward a definition”. Proceedings CHI. May 7-12, Vancouver, Canada. ISBN: 9781450302685 http://gamification-research.org/wp-content/uploads/2011/04/02-Deterding-Khaled-Nacke-Dixon.pdf

Domínguez-Martín, Eva (2015). “Periodismo inmersivo o cómo la realidad virtual y el videojuego influyen en la interfaz e interactividad del relato de actualidad”. El profesional de la información, v. 24, n. 4, pp. 413-423. https://doi.org/10.3145/epi.2015.jul.08

Duchowski, Andrew (2013). Eye tracking methodology: Theory and practice. London: Springer-Verlag. ISBN: 9781 846286087

Ferguson, Christopher J. (2011). "Video games and youth violence: A prospective analysis in adolescents". Journal of youth and adolescence, v. 40, n. 4, pp. 377-391.

https://doi.org/10.1007/s10964-010-9610-x

Frasca, Gonzalo (2007). Play the message. Play, game and videogame rhetoric. Copenhagen: IT University of Copenhagen. https://www.yumpu.com/en/document/read/11225449/frasca-play-the-message-phd/30

García-Ortega, Alba; García-Avilés, José-Alberto (2018). “Los newsgames como estrategia narrativa en el periodismo transmedia: propuesta de un modelo de análisis”. Revista mediterránea de comunicación, v. 9, n. 1, pp. 327-346.

https://doi.org/10.14198/MEDCOM2018.9.1.19

Girard, Charles; Ecalle, Jean; Magnan, Annie (2013). "Serious games as new educational tools: How effective are they? A meta-analysis of recent studies". Journal of computer assisted learning, v. 29, n. 3, pp. 207-219.

https://doi.org/10.1111/j.1365-2729.2012.00489.x

Gómez-García, Salvador; Cabeza-San-Deogracias, José (2016). “El discurso informativo de los newsgames: el caso Bárcenas en los juegos para dispositivos móviles". Cuadernos.info, n. 38, pp. 137-148.

https://doi.org/10.7764/cdi.38.593

Gómez-García, Salvador; Navarro-Sierra, Nuria (2013). “Videojuegos e información. Una aproximación a los newsgames españoles como nueva óptica informativa”. Icono 14, v. 11, n. 2, pp. 31-51.

https://doi.org/10.7195/ri14.v11i2.604

Goodrich, Kendall (2011). "Anarchy of effects? Exploring attention to online advertising and multiple outcomes". Psychology \& marketing, v. 28, n. 4, pp. 417-440.

https://doi.org/10.1002/mar.20371

Jenkins, Henry (2003). "Transmedia storytelling. Moving characters from books to films to video games can make them stronger and more compelling". MIT technology review, January 15.

https://www.technologyreview.com/s/401760/transmedia-storytelling

Jenkins, Henry (2006). Convergence culture: where old and new media collide. New York: New York University Press. ISBN: 9780814742952

Loftus, Geoffrey R.; Kallman, Howard J. (1979). "Encoding and use of detail information in picture recognition". Journal of experimental psychology: Human learning and memory, v. 5, n. 3, pp. 197-211.

https://doi.org/10.1037/0278-7393.5.3.197

Lugmayr, Artur; Sutinen, Erkki; Suhonen, Jarkko; Islas-Sedano, Carolina; Hlavacs, Helmut; Suero-Montero, Calkin (2017). "Serious storytelling - a first definition and review". Multimedia tools \& applications, v. 76, n. 14, pp. 15707-15733.

https://doi.org/10.1007/s11042-016-3865-5

Madan, Christopher R. (2010). “Neuromarketing: The next step in market research?”. Eureka, v. 1, n. 1, pp. 34-42. https://journals.library.ualberta.ca/eureka/index.php/eureka/article/view/7786/6493

Marcos-Recio, Juan-Carlos; Edo-Bolós, Concha; Parra-Valcarce, David (2018). “Remaining challenges for digital newspapers regarding informative updates: case studies in the Spanish media". Communication \& society, v. 31, n. 2, pp. 51-70. https://doi.org/10.15581/003.31.2.51-69

Papoutsi, Chara; Drigas, Athanasios S. (2016). "Games for empathy for social impact". International journal of engineering pedagogy, v. 6, n. 4, pp. 36-40.

https://doi.org/10.3991/ijep.v6i4.6064

Pérez-Seijo, Sara (2017). "Immersive journalism: From audience to first-person experience of news". In: Campos-Freire, Francisco; Rúas-Araujo, Xosé; Martínez-Fernández, Valentín; López-García, Xosé. Media and metamedia management. Cham: Springer, pp. 113-119. ISBN: 9783319460666

https://doi.org/10.1007/978-3-319-46068-0_14 
Ruiz-Collantes, Francisco-Xavier (2008). “Juegos y relatos como vivencias narrativas”. En: Scolari, Carlos-Alberto. Homo videoludens. Vic: Eumo, pp. 17-52. ISBN: 9788469568521

http://www.articaonline.com/wp-content/uploads/2014/02/Homo-Videoludens-2-0-De-Pacman-a-la-gamification.pdf

Sacco, Vittoria; Gorin, Valérie; Schiau, Nicolae (2018). "Immersive journalism and the migrant crisis: The case of Exils as a mobile radio reportaje". Journal of applied journalism \& media studies, v. 7, n. 1, pp. 197-213.

https://doi.org/10.1386/ajms.7.1.197_1

Scolari, Carlos-Alberto (2009). "Transmedia storytelling: Implicit consumers, narrative worlds, and branding in contemporary media production". International journal of communication, n. 3, pp. 586-606.

http://ijoc.org/index.php/ijoc/article/view/477

Scolari, Carlos-Alberto (2013). Homo videoludens 2.0. De Pacman a la gamificación. Col·lecció Transmedia XXI. Laboratori de Mitjans Interactius. Barcelona: Universitat de Barcelona. ISBN: 9788469568521

http://www.articaonline.com/wp-content/uploads/2014/02/Homo-Videoludens-2-0-De-Pacman-a-la-gamification.pdf

Sundar, S. Syham; Kang, Jin; Oprean, Danielle (2017). "Being there in the midst of the story: How immersive journalism affects our perceptions and cognitions". Cyberpsychology behavior and social networking, v. 20, n. 11, pp. $672-682$.

https://doi.org/10.1089/cyber.2017.0271

Ulrich, Frank; Helms, Niels-Henrik (2017). “Creating evaluation profiles for games designed to be fun: An interpretive framework for serious game mechanics". Simulation \& gaming, v. 48, n. 5, pp. 695-714.

https://doi.org/10.1177/1046878117709841

Vázquez-Herrero, Jorge; López-García, Xosé (2016). “La gamificación en el periodismo a través del documental interactivo: estudio comparativo de Pirate Fishing, Réfugiés y Montelab". En: Marfil, J. Pedro; Römer, Max. Retos del periodismo para el ejercicio responsable y libre de la profesión. Madrid: Sociedad Española de Periodística; Universidad Camilo José Cela, pp. 217-232. ISBN: 9788495891693

https://cutt.ly/WwnbNBz

Vázquez-Herrero, Jorge; López-García, Xosé (2017). “El documental interactivo como formato en los medios audiovisuales: estudio de caso de RTVE y Al Jazeera”. Anàlisi. Quaderns de comunicació i cultura, n. 57, pp. 47-61.

https://doi.org/10.5565/rev/analisi.3100

Vázquez-Herrero, Jorge; Negreira-Rey, María-Cruz; Pereira-Fariña, Xosé (2017). "Contribuciones del documental interactivo a la renovación de las narrativas periodísticas: realidades y desafíos. Revista latina de comunicación social, n. 72 , pp. 397-414.

https://doi.org/10.4185/RLCS-2017-1171

Yam, Alpha; Russell-Bennett, Rebekah; Foth, Marcus; Mulcahy, Rory (2017). "How does serious M-game technology encourage low-income households to perform socially responsible behaviors?". Psychology \& marketing, v. 34, n. 4, pp. 394-409.

https://doi.org/10.1002/mar.20996

\section{Cronología de la Documentación Española}

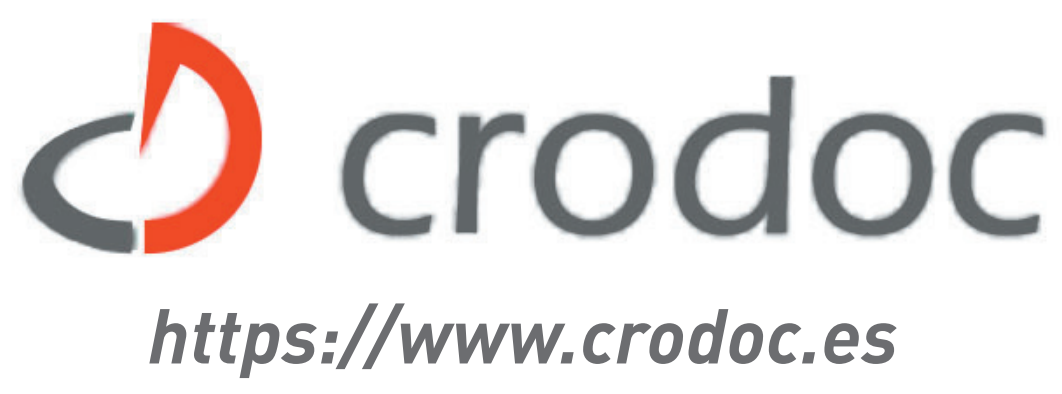

